\title{
Behaviour Analysis of Alloy Wheel Plant
}

\author{
Rajbala, Deepika Garg
}

\begin{abstract}
In this paper, Behaviour Analysis of an Alloy Wheel Plant utilizing RPGT under specific conditions has been discussed. An Alloy Wheel Plant is isolated into five sub-systems $P, Q, R, S$ and $T$ for instances of computations. An Alloy Wheel Plant consists of five frame woks for example Gravity Die Machine (P), Cutting Machine (Q), Solutiuonizing Chamber Machine (R), Azing Chamber Machine(S) and Shot Blasting Machine (T). These subsystems are associated in arrangement. On the off chance that any of the sub units comes up short, at that point the Alloy Wheel plant works in diminished state. In the event that at least two sub units fall flat, at that point systems comes up short. Parametric estimations of a system generally rely upon failure / repair rate of individual units. Single server fixes all sub-units. Framework parameters, for example, Availability, MTSF and Number of Server's Visits utilizing RPGT are determined. Specific cases and behaviour analysis w.r.t different rates are additionally completed pursue by graphs.
\end{abstract}

Keywords: Availability, busy-period of repairman, Behaviour Analysis

\section{INTRODUCTION}

Presently a day, makers need to create their items un interruptedly to satisfy the regularly expanding needs of the their items. They can do so as such by making their creation units as effective as could be allowed. This paper talks about behavior analysis of an alloy wheel plant separated into five sub framework. MTSF, busy period of server, Availability, Number of visits are determined to utilize RPGT. A benefit capacity is additionally characterized for analyzing the benefit of system. Kumar et. al. (2019) the goal behind this specific paper is, as a rule, to decide the criticality of various sub systems through the conducted research of a multi-state repairable strategy with warm repetition. The availability of the gadget is streamlined to evaluate the most extreme blends of failure just as fix rate parameters for various sub-systems. Kumar A., Garg D et al. (2019) have discussed on sensitivity analysis of a cold standby system with Priority for Preventive Maintenance. Gulati et. al (2018) this paper handles the exploration of unwavering quality strides of a two units unwanted program under various kinds of failure just as two sorts of maintenance. Kumar A., Goel P., Garg D., Sahu A. (2017) have likewise talked about on behavior analysis in the urea fertilizer industry. Manglik and Mangey (2016) the objective of the investigation paper is, to demonstrate the reliability strides of a unit by speaking to a modern program having three subsystems.

Revised Manuscript Received on December 30, 2019.

* Correspondence Author

Rajbala*, School of Basic and Applied Science, G.D. Goenka University (Gurgaon), Gurgaon, India.

Dr. Deepika Garg, School of Basic and Applied Science, G.D. Goenka University (Gurgaon), Kurukshetra, India.

(C) The Authors. Published by Blue Eyes Intelligence Engineering and Sciences Publication (BEIESP). This is an open access article under the CC BY-NC-ND license (http://creativecommons.org/licenses/by-nc-nd/4.0/)
Two of the subsystems have reserve gadget despite the fact that the third one has $\mathrm{n}$ gadgets in parallel arrangement. The entire system can fall flat as a result of a failure in subsystems and because of the cataclysmic calamity.A.K Barak, S.K Chhillar (2013) distributed an exploration paper where they portrayed the assessment related with a parallel system with priority to fix over maintenance under subjective stuns.M. Ram, S. B. Singh et. al (2013) and A. Mehrtash et. al (2012) have Likewise talked about on reliability. Liu et al. (2011) analyzed the availability direct connected with a repairable procedure where reserve exchanged over to main is put through breakdown. The creators accepted the fix time span of administration station pursues four run of the mill distributions: blend, exponential, uniform and Gamma. Wu, C.H. and Zhang, Z.G. (2010) examined a progression of repairable framework which involves two non-unclear portions with one repairer, for finding the unfaltering quality attributes for the proportionate. Exhibited a paper where an enthalpy enumerating is associated with the solidifying technique of an emotional shape throwing in a structure tossing system. Sharma, R. and Sharma, G.C. (2015) in the rapidly creating development, faithful quality expects a basic activity in each and every industry from creation to the action of various systems. Thusly, it is a huge endeavor for the administration of the framework to keep up the steadfast quality and nature of their things.S.C. And Preeti (2010) Built up the steadfastness estimation of an arrangement system in which the section reliabilities are dark is considered. The perfect testing plan depends upon parameters that are normally dark. A back to back plan is presented and differentiated and both balanced designation and ideal allocation. Numerical assessments for a two-section arrangement system are given. The results demonstrate that the proposed consecutive inspecting plan is extraordinarily improved than balanced distribution and is practically perfect. Nourelfath et al. (2010) dissected a multi-state strategy with arranged assembling planning just as preventive upkeep. The creators in like manner proposed a procedure to evaluate the cost and furthermore the hours of preventive upkeep, little repair as well as common place assembling limit. Kumar et al. (2009) registered fluffy reliability just as fuzzy accessibility of the sequential practice in butter oil preparing plant under various kinds of repair and problems of sub system. Reliability assessment of industrial frameworks assumes a significant role for the clients and management, to enhance Frame work parameters standby units are utilized, repairable standby frameworks are significant for the notoriety of the industry. A repairman assumes a significant job to advance the framework parameters straightforwardly/ indirectly. In the event the administrations have pre-hand data that a particular unit needs more care in care in contrast with different units then the administrations can accomplish a great deal in their objectives. 


\section{Behaviour Analysis of Alloy Wheel Plant}

This paper manages conduct investigations of a system having five units particular $\mathrm{P}, \mathrm{Q}, \mathrm{R}, \mathrm{S}, \mathrm{T}$ organized in an arrangement. System bombs totally when both of the units $\mathrm{P}, \mathrm{Q}, \mathrm{R}, \mathrm{S}$, or $\mathrm{T}$ comes up short. Taking consistent and factually autonomous failure and repair rate for every one of the units, system transition state diagram explaining all conceivable states, directed way transition along with disappointment and repair rates in transiting from one state to other utilizing Markov process and is displayed utilizing RPGT for behaviour analysis of framework parameters. Behaviour analysis and conclusion are drawn by planning tables and charts for various estimations of repair and disappointment paces of the units.

\section{ASSUMPTIONS AND NOTATIONS}

1. Single repair facility is accessible.

2. Failures and repairs are measurably free.

3. Repair is impeccable for example it doesn't harm any sub units during fix.
4. Whenever at least subunits flop at that point subsystem is in bombed state.

5. System is talked about for consistent state conditions.

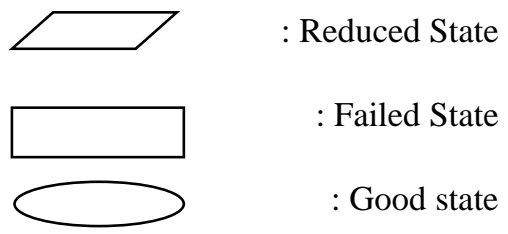

$\alpha_{i} / \beta_{i} \quad(1 \leq i \geq 6)$ : Constant failure/repair rates of units $\mathrm{A} /$ of unit B.

$\overline{\mathrm{P}}$ : Reduced state.

P, Q, R, S, T / p, q, r, s, t: Operative state / failed state.

Following the above assumption \& notations the Transition Diagram of the system is given in Fig. 1

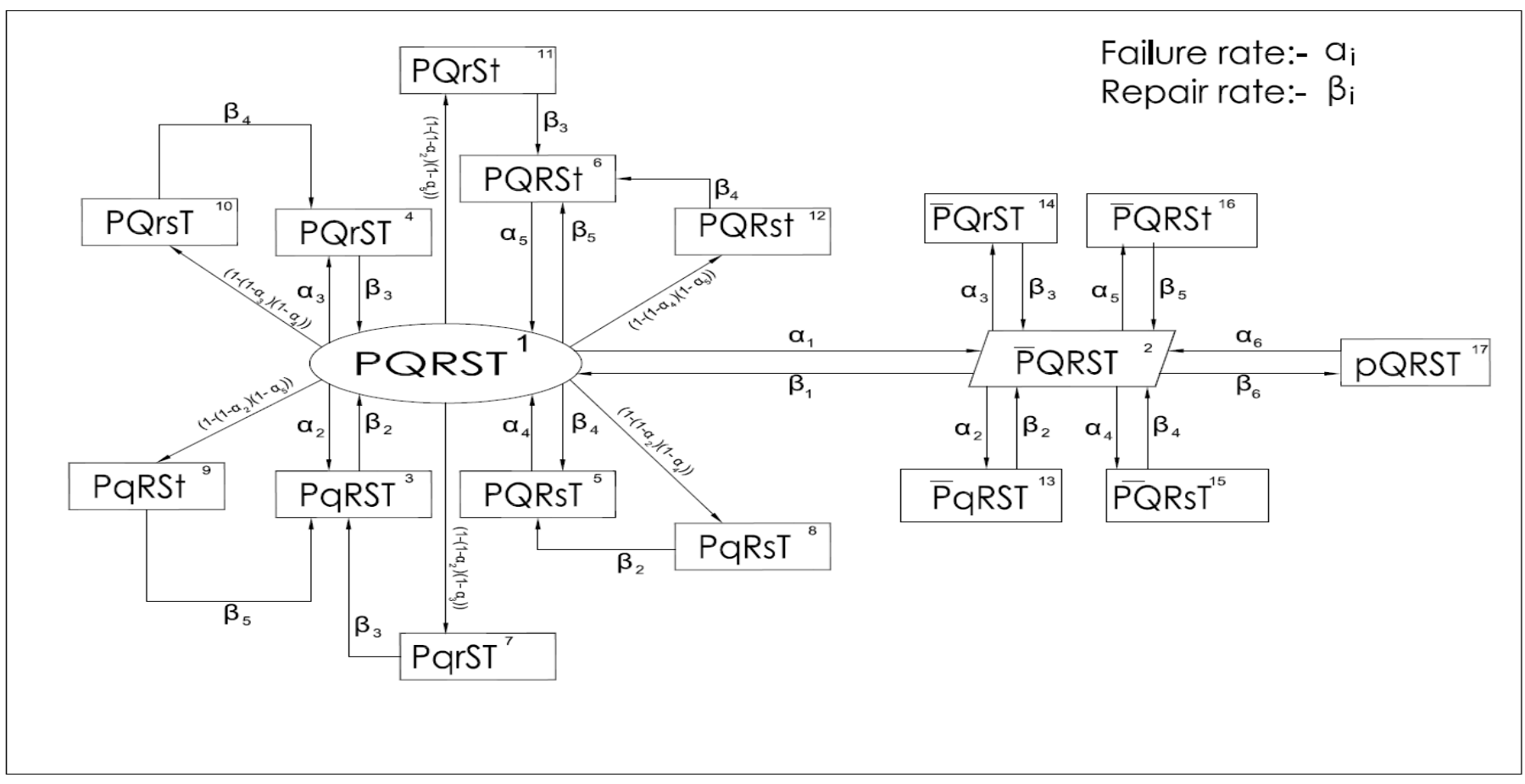

Fig.1. Transition Diagram of the System Design

\section{III.MODEL DESCRIPTION}

Here, a system which has five subsystems configured namely P, Q, R, S and T in series is modelled \& analyzed for system parameters. Subsystems P, Q, R, and S have components in series, so if any of the components fails leads that unit to failed state causing whole system to failed state. Failure of any one of these subsystems causes the complete failure of system. Subsystem T consists of two units. One unit of subsystem $\mathrm{T}$ is active and other unit are in cold standby mode. It is assumed that system is repairable in both cases. Repairs are perfect and absolute, so after the repair every subsystem works as good as new. The failure \& repair rates for units have been taken constant for steady state to represent the system transition state diagram. 
Table.I Various Paths from vertices

\begin{tabular}{|c|c|c|c|c|c|c|c|c|c|}
\hline $\begin{array}{l}\text { Verte } \\
\mathrm{X} \\
\mathrm{I}\end{array}$ & $\mathrm{J}=1$ & $\mathrm{~J}=2$ & $\mathrm{~J}=3$ & $\mathrm{~J}=4$ & $\mathrm{~J}=5$ & $\mathrm{~J}=6$ & $\mathrm{~J}=7$ & $\mathrm{~J}=8$ & $\mathrm{~J}=9$ \\
\hline $\mathrm{J}=1$ & $\begin{array}{l}(1,2,1)(1,3,1)( \\
1,4,1) \\
(1,5,1)(1,6,1) \\
(1,7,3,1)(1,9,3, \\
1) \\
(1,8,5,1)(1,10, \\
4,1) \\
(1,11,6,1)(1,12 \\
, 6,1)\end{array}$ & $(1,2)$ & $(1,3)$ & $(1,4)$ & $(1,5)$ & $(1,6)$ & $(1,7)$ & $(1,8)$ & $(1,9)$ \\
\hline $\mathrm{J}=2$ & $(2,1)$ & $\begin{array}{l}(2,1,2)(2,13,2) \\
(2,14,2)(2,15,2) \\
(2,16,2)(2,17,2)\end{array}$ & $(2,1,3)$ & $(2,1,4)$ & $(2,1,5)$ & $(2,1,6)$ & $(2,1,7)$ & $(2,1,8)$ & $(2,1,9)$ \\
\hline $\mathrm{J}=3$ & $(3,1)$ & $(3,1,2)$ & $(3,1,3)$ & $(3,1,4)$ & $(3,1,5)$ & $(3,1,6)$ & $(3,1,7)$ & $(3,1,8)$ & $(3,1,9)$ \\
\hline $\mathrm{J}=4$ & $(4,1)$ & $(4,1,2)$ & $(4,1,3)$ & $(4,1,4)$ & $(4,1,5)$ & $(4,1,6)$ & $(4,1,7)$ & $(4,1,8)$ & $(4,1,9)$ \\
\hline $\mathrm{J}=5$ & $(5,1)$ & $(5,1,2)$ & $(5,1,3)$ & $(5,1,4)$ & $(5,1,5)$ & $(5,1,6)$ & $(5,1,7)$ & $(5,1,8)$ & $(5,1,9)$ \\
\hline $\mathrm{J}=6$ & $(6,1)$ & $(6,1,2)$ & $(6,1,3)$ & $(6,1,4)$ & $(6,1,5)$ & $(6,1,6)$ & $(6,1,7)$ & $(6,1,8)$ & $(6,1,9)$ \\
\hline $\mathrm{J}=7$ & $(7,3,1)$ & $(7,3,1,2)$ & $(7,3)$ & $(7,3,1,4)$ & $(7,3,1,5)$ & $(7,3,1,6)$ & $(7,3,1,7)$ & $(7,3,1,8)$ & $(7,3,1,9)$ \\
\hline $\mathrm{J}=8$ & $(8,5,1)$ & $(8,5,1,2)$ & $(8,5,1,3)$ & $(8,5,1,4)$ & $(8,5,1,5)$ & $(8,5,1,6)$ & $(8,5,1,6)$ & $(8,5,1,7)$ & $(8,5,1,8)$ \\
\hline $\mathrm{J}=9$ & $(9,3,1)$ & $(9,3,1,2)$ & $(9,3)$ & $(9,3,1,4)$ & $(9,3,1,5)$ & $(9,3,1,6)$ & $(9,3,1,7)$ & $(9,3,1,8)$ & $(9,3,1,9)$ \\
\hline $\mathrm{J}=10$ & $(10,4,1)$ & $(10,4,1,2)$ & $\begin{array}{l}(10,4,1,3 \\
)\end{array}$ & $(10,4)$ & $\begin{array}{l}(10,4,1,5 \\
)\end{array}$ & $\begin{array}{l}(10,4,1,6 \\
)\end{array}$ & $\begin{array}{l}(10,4,1,7 \\
)\end{array}$ & $\begin{array}{l}(10,4,1,8 \\
)\end{array}$ & $\begin{array}{l}(10,4,1,9 \\
)\end{array}$ \\
\hline $\mathrm{J}=11$ & $(11,6,1)$ & $(11,6,1,2)$ & $\begin{array}{l}(11,6,1,3 \\
)\end{array}$ & $\begin{array}{l}(11,6,1,4 \\
)\end{array}$ & $\begin{array}{l}(11,6,1,5 \\
)\end{array}$ & $(11,6)$ & $\begin{array}{l}(11,6,1,7 \\
)\end{array}$ & $\begin{array}{l}(11,6,1,8 \\
)\end{array}$ & $(11,6,19)$ \\
\hline $\mathrm{J}=12$ & $(12,6,1)$ & $(12,6,1,2)$ & $\begin{array}{l}(1- \\
2,6,1,3)\end{array}$ & $\begin{array}{l}(12,6,1,4 \\
)\end{array}$ & $\begin{array}{l}(12,6,1,5 \\
)\end{array}$ & $(12,6)$ & $\begin{array}{l}(12,6,1,7 \\
)\end{array}$ & $\begin{array}{l}(12,6,1,8 \\
)\end{array}$ & $\begin{array}{l}(12,6,1,9 \\
)\end{array}$ \\
\hline $\mathrm{J}=13$ & $(13,2,1)$ & $(13,2)$ & $\begin{array}{l}(13,2,1,3 \\
)\end{array}$ & $\begin{array}{l}(13,2,1,4 \\
)\end{array}$ & $\begin{array}{l}(13,2,1,5 \\
)\end{array}$ & $\begin{array}{l}(13,2,1,6 \\
)\end{array}$ & $\begin{array}{l}(13,2,1,7 \\
)\end{array}$ & $\begin{array}{l}(13,2,1,8 \\
)\end{array}$ & $\begin{array}{l}(13,2,1,9 \\
)\end{array}$ \\
\hline $\mathrm{J}=14$ & $(14,2,1)$ & $(14,2)$ & $\begin{array}{l}(14,2,1,3 \\
)\end{array}$ & $\begin{array}{l}(14,2,1,4 \\
)\end{array}$ & $\begin{array}{l}(14,2,1,5 \\
)\end{array}$ & $\begin{array}{l}(14,2,1,6 \\
)\end{array}$ & $\begin{array}{l}(14,2,1,7 \\
)\end{array}$ & $\begin{array}{l}(14,2,1,8 \\
)\end{array}$ & $\begin{array}{l}(14,2,1,9 \\
)\end{array}$ \\
\hline $\mathrm{J}=15$ & $(15,2,1)$ & $(15,2)$ & $\begin{array}{l}(15,2,1,3 \\
)\end{array}$ & $\begin{array}{l}(15,2,1,4 \\
)\end{array}$ & $\begin{array}{l}(15,2,1,5 \\
)\end{array}$ & $\begin{array}{l}(15,2,1,6 \\
)\end{array}$ & $\begin{array}{l}(15,2,1,7 \\
)\end{array}$ & $\begin{array}{l}(15,2,1,8 \\
)\end{array}$ & $\begin{array}{l}(15,2,1,9 \\
)\end{array}$ \\
\hline $\mathrm{J}=16$ & $(16,2,1)$ & $(16,2)$ & $\begin{array}{l}(16,2,1,3 \\
)\end{array}$ & $\begin{array}{l}(16,2,1,4 \\
)\end{array}$ & $\begin{array}{l}(16,2,1,5 \\
)\end{array}$ & $\begin{array}{l}(16,2,1,6 \\
)\end{array}$ & $\begin{array}{l}(16,2,1,7 \\
)\end{array}$ & $\begin{array}{l}(16,2,1,8 \\
)\end{array}$ & $(16,2,19)$ \\
\hline $\mathrm{J}=17$ & $(17,2,1)$ & $(17,2)$ & $\begin{array}{l}(17,2,1,3 \\
)\end{array}$ & $\begin{array}{l}(17,2,1,4 \\
)\end{array}$ & $\begin{array}{l}(17,2,1,5 \\
)\end{array}$ & $\begin{array}{l}(17,2,1,6 \\
)\end{array}$ & $\begin{array}{l}(17,2,1,7 \\
)\end{array}$ & $\begin{array}{l}(17,2,1,8 \\
)\end{array}$ & $\begin{array}{l}(17,2,1,9 \\
)\end{array}$ \\
\hline
\end{tabular}

\begin{tabular}{|l|l|l|l|l|l|l|l|l|l|}
\hline $\begin{array}{c}\text { Verte } \\
\mathrm{X} \\
\mathrm{I}\end{array}$ & $\mathrm{J}=9$ & $\mathrm{~J}=10$ & $\mathrm{~J}=11$ & $\mathrm{~J}=12$ & $\mathrm{~J}=13$ & $\mathrm{~J}=14$ & $\mathrm{~J}=15$ & $\mathrm{~J}=16$ \\
$\mathrm{~J}=1$ & $(1,9)$ & $(1,10)$ & $(1,11)$ & $(1,12)$ & $(1,2,13)$ & $(1,2,14)$ & $(1,2,15)$ & $(1,2,16)$ & $(1,2,17)$ \\
\hline $\mathrm{J}=2$ & $(2,1,9)$ & $(2,1,10)$ & $(2,1,11)$ & $(2,1,12)$ & $(2,13)$ & $(2,14)$ & $(2,15)$ & $(2,16)$ & $(2,17)$ \\
\hline
\end{tabular}


Behaviour Analysis of Alloy Wheel Plant

\begin{tabular}{|c|c|c|c|c|c|c|c|c|c|}
\hline $\mathrm{J}=3$ & $(3,1,9)$ & $(3,1,10)$ & $(3,1,11)$ & $(3,1,12)$ & $(3,1,2,13)$ & $(3,1,2,14)$ & $(3,1,2,15)$ & $(3,1,2,16)$ & $(3,1,2,17)$ \\
\hline $\mathrm{J}=4$ & $(4,1,9)$ & $(4,1,10)$ & $(4,1,11)$ & $(4,1,12)$ & $(4,1,2,13)$ & $(4,1,2,14)$ & $(4,1,2,15)$ & $(4,1,2,16)$ & $(4,1,2,17)$ \\
\hline $\mathrm{J}=5$ & $(5,1,9)$ & $(5,1,10)$ & $(5,1,11)$ & $(5,1,12)$ & $(5,1,2,13)$ & $(5,1,2,14)$ & $(5,1,2,15)$ & $(5,1,2,16)$ & $(5,1,2,17)$ \\
\hline $\mathrm{J}=6$ & $(6,1,9)$ & $(6,1,10)$ & $(6,1,11)$ & $(6,1,12)$ & $(6,1,2,13)$ & $(6,1,2,14)$ & $(6,1,2,15)$ & $(6,1,2,16)$ & $(6,1,2,17)$ \\
\hline $\mathrm{J}=7$ & $(7,3,1,9)$ & $(7,3,1,10)$ & $(7,3,1,11)$ & $(7,3,1,12)$ & $(7,3,1,2,13)$ & $(7,3,1,2,14)$ & $(7,3,1,2,15)$ & $(7,3,1,2,16)$ & $(7,3,1,2,17)$ \\
\hline $\mathrm{J}=8$ & $(8,5,1,9)$ & $(8,5,1,10)$ & $(8,5,1,11)$ & $(8,5,1,12)$ & $(8,5,1,2,13)$ & $(8,5,1,2,14)$ & $(8,5,1,2,15)$ & $(8,5,1,2,16)$ & $(8,5,1,2,17)$ \\
\hline $\mathrm{J}=9$ & $(9,3,1,9)$ & $(9,3,1,10)$ & $(9,3,1,11)$ & $(9,3,1,12)$ & $(9,3,1,2,13)$ & $(9,3,1,2,14)$ & $(9,3,1,2,15)$ & $(9,3,1,2,16)$ & $(9,3,1,2,17)$ \\
\hline $\mathrm{J}=10$ & $\begin{array}{l}(10,4,1,9 \\
)\end{array}$ & $\begin{array}{l}(10,4,1,10 \\
)\end{array}$ & $\begin{array}{l}(10,4,1,11 \\
)\end{array}$ & $\begin{array}{l}(10,4,1,12 \\
)\end{array}$ & $\begin{array}{l}(10,4,1,2,13 \\
)\end{array}$ & $\begin{array}{l}\text { (10,4,1,2,1 } \\
\text { 4) }\end{array}$ & $\begin{array}{l}(10,4,1,2,1 \\
5)\end{array}$ & $\begin{array}{l}(10,4,1,2,1 \\
6)\end{array}$ & $\begin{array}{l}(10,4,1,2,17 \\
\end{array}$ \\
\hline $\mathrm{J}=11$ & $\begin{array}{l}(11,6,1,9 \\
)\end{array}$ & $\begin{array}{l}(11,6,1,10 \\
)\end{array}$ & $\begin{array}{l}(11,6,1,11 \\
)\end{array}$ & $\begin{array}{l}(11,6,1,12 \\
)\end{array}$ & $\begin{array}{l}(11,6,1,2,13 \\
)\end{array}$ & $\begin{array}{l}(11,6,1,2,1 \\
4)\end{array}$ & $\begin{array}{l}(11,6,1,2,1 \\
5)\end{array}$ & $\begin{array}{l}\text { (11,6,1,2,1 } \\
6)\end{array}$ & $\begin{array}{l}(11,6,1,2,17 \\
\text { (1) }\end{array}$ \\
\hline $\mathrm{J}=12$ & $\begin{array}{l}(12,6,1,9 \\
)\end{array}$ & $\begin{array}{l}(12,6,1,10 \\
)\end{array}$ & $\begin{array}{l}(12,6,1,11 \\
)\end{array}$ & $\begin{array}{l}(12,6,1,12 \\
)\end{array}$ & $\begin{array}{l}(12,6,1,2,13 \\
)\end{array}$ & $\begin{array}{l}(12,6,1,2,1 \\
4)\end{array}$ & $\begin{array}{l}\text { (12,6,1,2,1 } \\
5)\end{array}$ & $\begin{array}{l}(12,6,1,2,1 \\
6)\end{array}$ & $\begin{array}{l}(12,6,1,2,17 \\
l^{2}\end{array}$ \\
\hline $\mathrm{J}=13$ & $\begin{array}{l}(13,2,1,9 \\
)\end{array}$ & $\begin{array}{l}(13,2,1,10 \\
)\end{array}$ & $\begin{array}{l}(13,2,1,11 \\
)\end{array}$ & $\begin{array}{l}(13,2,1,12 \\
)\end{array}$ & $(13,2,13)$ & $(13,2,14)$ & $(13,2,15)$ & $(13,2,16)$ & $(13,2,17)$ \\
\hline $\mathrm{J}=14$ & $\begin{array}{l}(14,2,1,9 \\
)^{2}\end{array}$ & $\begin{array}{l}(14,2,1,10 \\
)\end{array}$ & $\begin{array}{l}(14,2,1,11 \\
)\end{array}$ & $\begin{array}{l}(14,2,1,12 \\
)\end{array}$ & $(14,2,13)$ & $(14,2,14)$ & $(14,2,15)$ & $(14,2,16)$ & $(14,2,17)$ \\
\hline $\mathrm{J}=15$ & $\begin{array}{l}(15,2,1,9 \\
)\end{array}$ & $\begin{array}{l}(15,2,1,10 \\
)\end{array}$ & $\begin{array}{l}(15,2,1,11 \\
)\end{array}$ & $\begin{array}{l}(15,2,1,12 \\
)\end{array}$ & $(15,2,13)$ & $(15,2,14)$ & $(15,2,15)$ & $(15,2,16)$ & $(15,2,17)$ \\
\hline $\mathrm{J}=16$ & $\begin{array}{l}(16,2,1,9 \\
)\end{array}$ & $\begin{array}{l}(16,2,1,10 \\
)\end{array}$ & $\begin{array}{l}(16,2,1,11 \\
)\end{array}$ & $\begin{array}{l}(16,2,1,12 \\
)\end{array}$ & $(16,2,13)$ & $(16,2,14)$ & $(13,2,15)$ & $(16,2,16)$ & $(16,2,17)$ \\
\hline $\mathrm{J}=17$ & $\begin{array}{l}(17,2,1,9 \\
)\end{array}$ & $\begin{array}{l}(17,2,1,10 \\
)\end{array}$ & $\begin{array}{l}(17,2,1,11 \\
)\end{array}$ & $\begin{array}{l}(17,2,1,12 \\
)\end{array}$ & $(17,2,13)$ & $(17,2,14)$ & $(17,2,15)$ & $(17,2,16)$ & $(17,2,17)$ \\
\hline
\end{tabular}

Table II. Primary, Secondary, Tertiary Circuits w. r. t. the Simple Paths (Initial-State '1') To Vertex '1'

\begin{tabular}{|l|l|l|l|l|l|}
\hline Vertex & \multicolumn{1}{|c|}{$\mathrm{S}_{\mathrm{R}}$} \\
\hline
\end{tabular}




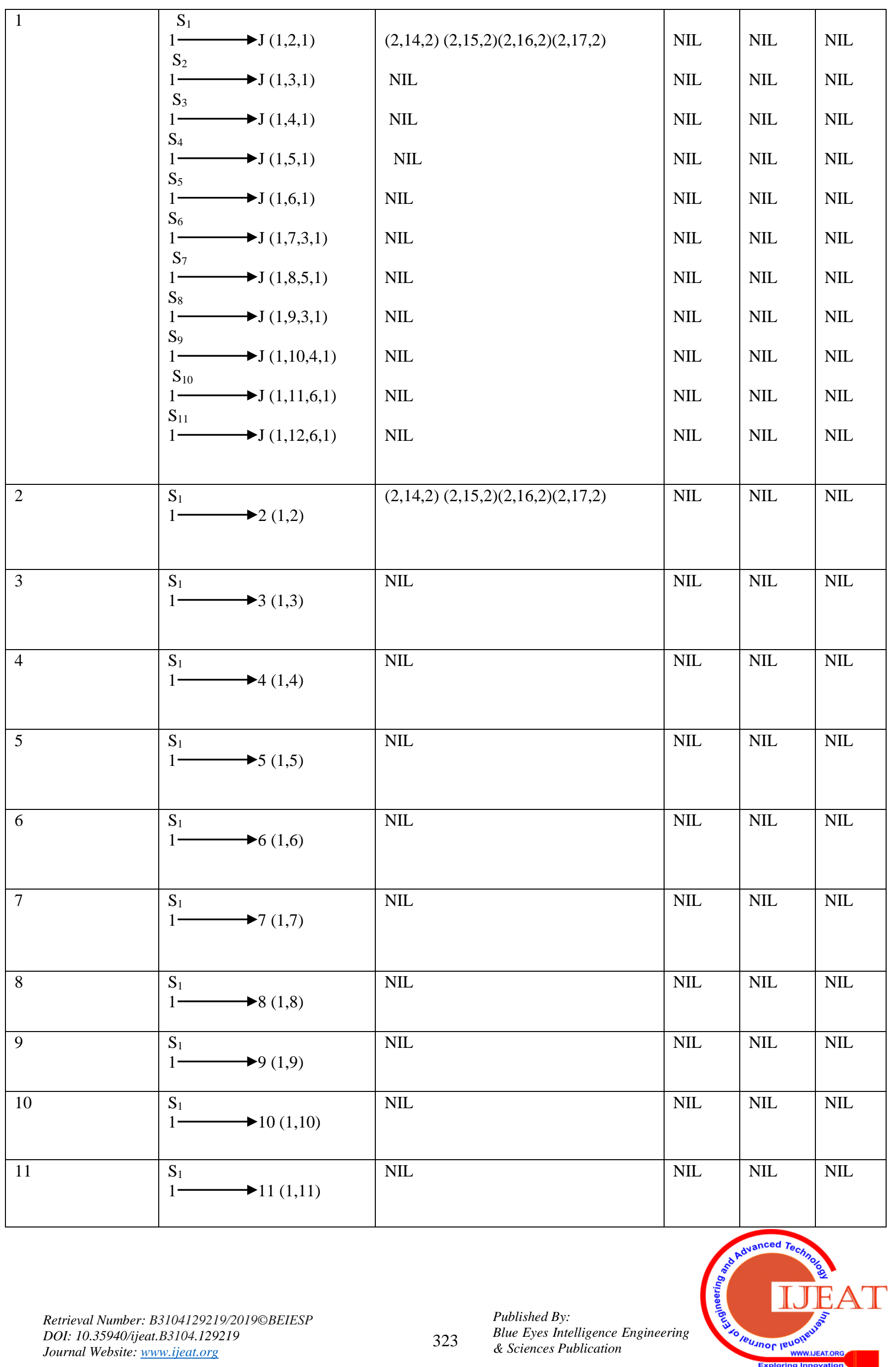


Behaviour Analysis of Alloy Wheel Plant

\begin{tabular}{|c|c|c|c|c|c|}
\hline 12 & $\begin{array}{l}\mathrm{S}_{1} \\
1 \longrightarrow 12(1,2)\end{array}$ & NIL & NIL & NIL & NIL \\
\hline 13 & $\begin{array}{l}\mathrm{S}_{1} \\
1 \longrightarrow 13(1,2,13)\end{array}$ & $(2,14,2)(2,15,2)(2,16,2)(2,17,2)$ & NIL & NIL & NIL \\
\hline 14 & $\begin{array}{l}\mathrm{S}_{1} \\
1 \longrightarrow 14(1,2,14)\end{array}$ & $(2,13,2)(2,15,2)(2,16,2)(2,17,2)$ & NIL & NIL & NIL \\
\hline 15 & $\begin{array}{l}\mathrm{S}_{1} \\
1 \longrightarrow 15(1,2,15)\end{array}$ & $(2,14,2)(2,13,2)(2,16,2)(2,17,2)$ & NIL & NIL & NIL \\
\hline 16 & $\begin{array}{l}\mathrm{S}_{1} \\
1 \longrightarrow 16(1,2,16)\end{array}$ & $(2,14,2)(2,15,2)(2,13,2)(2,17,2)$ & NIL & NIL & NIL \\
\hline 17 & $\begin{array}{l}\mathrm{S}_{1} \\
1 \longrightarrow 17(1,2,17)\end{array}$ & $(2,14,2)(2,15,2)(2,16,2)(2,13,2)$ & NIL & NIL & NIL \\
\hline
\end{tabular}

Table III. Transition Probabilities

\begin{tabular}{|c|c|}
\hline $\mathrm{q}_{\mathrm{i}, \mathrm{j}}(\mathrm{t})$ & $\mathrm{P}_{\mathrm{i}, \mathrm{j}}=\mathrm{q}_{\mathrm{i}, \mathrm{j}}{ }^{*}(\mathrm{t})$ \\
\hline $\mathrm{Q}_{1,2}(\mathrm{t})=\alpha_{1} e^{-\left(\alpha_{1}+\alpha_{2}+\alpha_{3}+\alpha_{4}+\alpha_{5}+\alpha_{6}+\cdots+\alpha_{12}\right)(t)}$ & $\mathrm{P}_{1,2}(\mathrm{t})=\frac{\alpha_{1}}{(\alpha+\alpha+\alpha+\alpha+\alpha+\alpha+\alpha)}$ \\
\hline $\mathrm{Q}_{1,3}(\mathrm{t})=\alpha_{2} e^{-\left(\alpha_{1}+\alpha_{2}+\alpha_{3}+\alpha_{4}+\alpha_{5}+\alpha_{6}+\cdots+\alpha_{12}\right)(t)}$ & 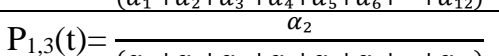 \\
\hline $\mathrm{Q}_{1,4}(\mathrm{t})=\alpha_{3} e^{-\left(\alpha_{1}+\alpha_{2}+\alpha_{3}+\alpha_{4}+\alpha_{5}+\alpha_{6}+\cdots+\alpha_{12}\right)(t)}$ & $\mathrm{P}_{1,4}(\mathrm{t})=\frac{\alpha_{3}}{\left(\alpha_{1}+\alpha_{2}+\alpha_{2}+\alpha_{1}+\alpha_{-}+\alpha_{1}+\cdots+\alpha_{1}\right)}$ \\
\hline $\mathrm{Q}_{1,5}(\mathrm{t})=\alpha_{4} e^{-\left(\alpha_{1}+\alpha_{2}+\alpha_{3}+\alpha_{4}+\alpha_{5}+\alpha_{6}+\cdots+\alpha_{12}\right)(t)}$ & 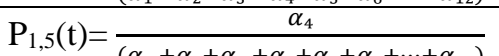 \\
\hline $\mathrm{Q}_{1,6}(\mathrm{t})=\alpha_{5} e^{-\left(\alpha_{1}+\alpha_{2}+\alpha_{3}+\alpha_{4}+\alpha_{5}+\alpha_{6}+\cdots+\alpha_{12}\right)(t)}$ & $\mathrm{P}_{1,6}(\mathrm{t})=\frac{\alpha_{5}}{\alpha_{5}+\alpha_{12}+\alpha_{12}}$ \\
\hline $\mathrm{Q}_{1,7}(\mathrm{t})=\alpha_{7} e^{-\left(\alpha_{1}+\alpha_{2}+\alpha_{3}+\alpha_{4}+\alpha_{5}+\alpha_{6}+\cdots+\alpha_{12}\right)(t)}$ & $\mathrm{P}_{1,7}(\mathrm{t})=\frac{\alpha_{7}}{\alpha_{7}}$ \\
\hline $\mathrm{Q}_{1,8}(\mathrm{t})=\alpha_{8} e^{-\left(\alpha_{1}+\alpha_{2}+\alpha_{3}+\alpha_{4}+\alpha_{5}+\alpha_{6}+\cdots+\alpha_{12}\right)(t)}$ & $\mathrm{P}_{1,8}(\mathrm{t})=\frac{\alpha_{8}}{\left(\alpha_{1}+\alpha_{2}+\alpha_{3}+\alpha_{1}+\alpha_{5}+\alpha_{1}+\cdots+\alpha_{10}\right)}$ \\
\hline $\mathrm{Q}_{1,9}(\mathrm{t})=\alpha_{9} e^{-\left(\alpha_{1}+\alpha_{2}+\alpha_{3}+\alpha_{4}+\alpha_{5}+\alpha_{6}+\cdots+\alpha_{12}\right)(t)}$ & $\mathrm{P}_{1,9}(\mathrm{t})=\frac{\alpha_{9}}{\left(\alpha_{1}+\alpha_{2}+\alpha_{3}+\alpha_{1}+\alpha_{5}+\alpha_{1}+\cdots+\alpha_{10}\right)}$ \\
\hline $\mathrm{Q}_{1,10}(\mathrm{t})=\alpha_{10} e^{-\left(\alpha_{1}+\alpha_{2}+\alpha_{3}+\alpha_{4}+\alpha_{5}+\alpha_{6}+\cdots+\alpha_{12}\right)(t)}$ & $\mathrm{P}_{1,10}(\mathrm{t})=\frac{\alpha_{10}}{\alpha^{2}}$ \\
\hline $\mathrm{Q}_{1,11}(\mathrm{t})=\alpha_{11} e^{-\left(\alpha_{1}+\alpha_{2}+\alpha_{3}+\alpha_{4}+\alpha_{5}+\alpha_{6}+\cdots+\alpha_{12}\right)(t)}$ & $\mathrm{P}_{1,11}(\mathrm{t})=\frac{\left.\alpha_{1}+a_{2}+u_{3}+a_{4}+a_{5}+u_{6}+\cdots+u_{12}\right)}{\alpha_{11}}$ \\
\hline $\mathrm{Q}_{1,12}(\mathrm{t})=\alpha_{12} e^{-\left(\alpha_{1}+\alpha_{2}+\alpha_{3}+\alpha_{4}+\alpha_{5}+\alpha_{6}+\cdots+\alpha_{12}\right)(t)}$ & $\mathrm{P}_{112}(\mathrm{t})=\frac{\left(\alpha_{1}+\alpha_{2}+\alpha_{3}+\alpha_{4}+\alpha_{5}+\alpha_{6}+\cdots+\alpha_{12}\right)}{\alpha_{12}}$ \\
\hline $\mathrm{Q}_{2,1}(\mathrm{t})=\beta_{1} e^{-\left(\beta_{1}+\alpha_{2}+\alpha_{3}+\alpha_{4}+\alpha_{5}+\alpha_{6}\right)(t)}$ & $P_{1}(t)=\frac{\left(\alpha_{1}+\alpha_{2}+\alpha_{3}+\alpha_{4}+\alpha_{5}+\alpha_{6}+\cdots+\alpha_{12}\right)}{\beta_{1}}$ \\
\hline & ${ }_{2,1(1)-}\left(\beta_{1}+\alpha_{2}+\alpha_{3}+\alpha_{4}+\alpha_{5}+\alpha_{6}\right)$ \\
\hline $\mathrm{Q}_{2,13}(\mathrm{t})=\alpha_{2} e^{-\left(\beta_{1}+\alpha_{2}+\alpha_{3}+\alpha_{4}+\alpha_{5}+\alpha_{6}\right)(t)}$ & $\mathrm{P}_{2,13}(\mathrm{t})=\frac{\alpha_{2}}{\left(\beta_{1}+\alpha_{2}+\alpha_{3}+\alpha_{4}+\alpha_{5}+\alpha_{6}\right)}$ \\
\hline $\mathrm{Q}_{2,14}(\mathrm{t})=\alpha_{3} e^{-\left(\beta_{1}+\alpha_{2}+\alpha_{3}+\alpha_{4}+\alpha_{5}+\alpha_{6}\right)(t)}$ & $\mathrm{P}_{2,14}(\mathrm{t})=\frac{\alpha_{3}}{\left(\beta_{1}+\alpha_{2}+\alpha_{3}+\alpha_{4}+\alpha_{5}+\alpha_{6}\right)}$ \\
\hline $\mathrm{Q}_{2,15}(\mathrm{t})=\alpha_{4} e^{-\left(\beta_{1}+\alpha_{2}+\alpha_{3}+\alpha_{4}+\alpha_{5}+\alpha_{6}\right)(t)}$ & $\mathrm{P}_{2,15}(\mathrm{t})=\frac{\alpha_{4}}{\left(\beta_{1}+\alpha_{2}+\alpha_{2}+\alpha_{4}+\alpha_{-}+\alpha_{2}\right)}$ \\
\hline $\mathrm{Q}_{2,16}(\mathrm{t})=\alpha_{5} e^{-\left(\beta_{1}+\alpha_{2}+\alpha_{3}+\alpha_{4}+\alpha_{5}+\alpha_{6}\right)(t)}$ & $\mathrm{P}_{2,16}(\mathrm{t})=\frac{\alpha_{5}}{\rho^{2}}$ \\
\hline $\mathrm{Q}_{2,17}(\mathrm{t})=\alpha_{6} \mathrm{e}^{-\left(\beta_{1}+\alpha_{2}+\alpha_{3}+\alpha_{4}+\alpha_{5}+\alpha_{6}\right)(t)}$ & $\mathrm{P}_{2,17}(\mathrm{t})=\frac{\left(p_{1}+u_{2}+u_{3}+a_{4}+u_{5}+u_{6}\right)}{\alpha_{6}}$ \\
\hline $\mathrm{Q}_{3,1}(\mathrm{t})=\beta_{2} e^{-\left(\beta_{2}\right)(t)}$ & $P_{3,1}(t)=1\left(\frac{\left.\beta_{2}+\alpha_{2}+\alpha_{3}+\alpha_{4}+\alpha_{5}+\alpha_{6}\right)}{\beta}\right)$ \\
\hline $\mathrm{Q}_{4,1}(\mathrm{t})=\beta_{3} e^{-\left(\beta_{3}\right)(t)}$ & $\mathrm{P}_{4,1}(\mathrm{t})=1$ \\
\hline $\mathrm{Q}_{5,1}(\mathrm{t})=\alpha_{4} e^{-\left(\alpha_{4}\right)(t)}$ & $P_{5,1}(t)=1$ \\
\hline $\mathrm{Q}_{6,1}(\mathrm{t})=\alpha_{5} e^{-\left(\alpha_{5}\right)(t)}$ & $\mathrm{P}_{6,1}(\mathrm{t})=1$ \\
\hline $\mathrm{Q}_{7,1}(\mathrm{t})=\alpha_{7} e^{-\left(\alpha_{7}\right)(t)}$ & $\mathrm{P}_{7,1}(\mathrm{t})=1$ \\
\hline $\mathrm{Q}_{8,1}(\mathrm{t})=\alpha_{8} e^{-\left(\alpha_{8}\right)(t)}$ & $\mathrm{P}_{8,1}(\mathrm{t})=1$ \\
\hline $\mathrm{Q}_{9,1}(\mathrm{t})=\alpha_{9} e^{-\left(\alpha_{9}\right)(t)}$ & $P_{9,1}(t)=1$ \\
\hline $\mathrm{Q}_{10,1}(\mathrm{t})=\alpha_{10} e^{-\left(\alpha_{10}\right)(t)}$ & $\mathrm{P}_{10,1}(\mathrm{t})=1$ \\
\hline $\mathrm{Q}_{11,1}(\mathrm{t})=\alpha_{11} e^{-\left(\alpha_{11}\right)(t)}$ & $P_{11,1}(t)=1$ \\
\hline
\end{tabular}




\begin{tabular}{|l|l|}
\hline $\mathrm{Q}_{12,1}(\mathrm{t})=\alpha_{12} e^{-\left(\alpha_{12}\right)(t)}$ & $\mathrm{P}_{12,1}(\mathrm{t})=1$ \\
\hline $\mathrm{Q}_{13,2}(\mathrm{t})=\beta_{13} e^{-\left(\beta_{2}\right)(t)}$ & $\mathrm{P}_{13,2}(\mathrm{t})=1$ \\
\hline $\mathrm{Q}_{14,2}(\mathrm{t})=\beta_{14} e^{-\left(\beta_{3}\right)(t)}$ & $\mathrm{P}_{14,2}(\mathrm{t})=1$ \\
\hline $\mathrm{Q}_{15,2}(\mathrm{t})=\beta_{15} e^{-\left(\beta_{4}\right)(t)}$ & $\mathrm{P} 15,2(\mathrm{t})=1$ \\
\hline $\mathrm{Q}_{16,2}(\mathrm{t})=\beta_{16} e^{-\left(\beta_{5}\right)(t)}$ & $\mathrm{P}_{16,2}(\mathrm{t})=1$ \\
\hline $\mathrm{Q}_{17,2}(\mathrm{t})=\beta_{17} e^{-\left(\beta_{6}\right)(t)}$ & $\mathrm{P}_{17,2}(\mathrm{t})=1$ \\
\hline
\end{tabular}

Table IV. Mean Sojourn Times

\begin{tabular}{|c|c|}
\hline $\mathrm{R}_{\mathrm{i}}(\mathrm{t})$ & $\mu_{\mathrm{i}} \mathrm{R}_{\mathrm{i}}^{*}(0)$ \\
\hline $\mathrm{R}_{1}(\mathrm{t})=\quad e^{-\left(\alpha_{1}+\alpha_{2}+\alpha_{3}+\alpha_{4}+\alpha_{5}+\alpha_{6}+\cdots+\alpha_{12}\right)(t)}$ & $\mu_{1}=\frac{1}{\left(\alpha_{1}+\alpha_{2}+\alpha_{3}+\alpha_{4}+\alpha_{5}+\alpha_{6}+\cdots+\alpha_{12}\right)}$ \\
\hline $\mathrm{R}_{2}(\mathrm{t})=e^{-\left(\beta_{1}+\alpha_{2}+\alpha_{3}+\alpha_{4}+\alpha_{5}+\alpha_{6}\right)(t)}$ & $\mu_{2}=\frac{1}{\left.\beta_{1}+\alpha_{2}+\alpha_{3}+\alpha_{4}+\alpha_{5}+\alpha_{6}\right)}$ \\
\hline $\mathrm{R}_{3}(\mathrm{t})=e^{-\beta_{2}(t)}$ & $\mu_{3}=\frac{1}{\beta_{2}}$ \\
\hline $\mathrm{R}_{4}(\mathrm{t})=e^{-\beta_{3}(t)}$ & $\mu_{4}=\frac{1}{\beta_{3}}$ \\
\hline $\mathrm{R}_{5}(\mathrm{t})=e^{-\alpha_{4}(t)}$ & $\mu_{5}=\frac{1}{\alpha_{4}}$ \\
\hline $\mathrm{R}_{6}(\mathrm{t})=e^{-\alpha_{5}(t)}$ & $\mu_{6}=\frac{1}{\alpha_{5}}$ \\
\hline $\mathrm{R}_{7}(\mathrm{t})=e^{-\alpha_{7}(t)}$ & $\mu_{7}=\frac{1}{\alpha_{7}}$ \\
\hline $\mathrm{R}_{8}(\mathrm{t})=e^{-\alpha_{8}(t)}$ & $\mu_{8}=\frac{1}{\alpha_{8}}$ \\
\hline $\mathrm{R}_{9}(\mathrm{t})=e^{-\alpha_{9}(t)}$ & $\mu_{9}=\frac{1}{\alpha_{9}}$ \\
\hline $\mathrm{R}_{10}(\mathrm{t})=e^{-\alpha_{10}(t)}$ & $\mu_{10}=\frac{1}{\alpha_{10}}$ \\
\hline $\mathrm{R}_{11}(\mathrm{t})=e^{-\alpha_{I I}(t)}$ & $\mu_{11}=\frac{1}{\alpha_{l 1}}$ \\
\hline $\mathrm{R}_{12}(\mathrm{t})=e^{-\alpha_{12}(t)}$ & $\mu_{12}=\frac{1}{\alpha_{12}}$ \\
\hline $\mathrm{R}_{13}(\mathrm{t})=e^{-\beta_{2}(t)}$ & $\mu_{13}=\frac{1}{\beta_{2}}$ \\
\hline $\mathrm{R}_{14}(\mathrm{t})=e^{-\beta_{3}(t)}$ & $\mu_{14}=\frac{1}{\beta_{3}}$ \\
\hline $\mathrm{R}_{15}(\mathrm{t})=e^{-\beta_{4}(t)}$ & $\mu_{15}=\frac{1}{\beta_{4}}$ \\
\hline $\mathrm{R}_{16}(\mathrm{t})=e^{-\beta_{5}(t)}$ & $\mu_{16}=\frac{1}{\beta_{s}}$ \\
\hline $\mathrm{R}_{17}(\mathrm{t})=e^{-\beta_{6}(t)}$ & $\mu_{17}=\frac{1}{\beta_{6}}$ \\
\hline
\end{tabular}

\section{ASSESSMENT OF PARAMETER}

The frame work as under the transition likelihood variable of all the reachable states from the base state ' 1 ' is: $\mathrm{V}_{1,1}=1$ (Verified) $\mathrm{V}_{1}$,

${ }_{2}=\frac{(1,2)}{(1-(2,13,2))(1-(2,14,2))(1-(2,15,2))(1-(2,16,2))(1-(2,17,2))}$ and So on.MTSF $\left(\mathbf{T}_{\mathbf{0}}\right)$ : From figure 1, the regenerative UN - failed stateto Which framework can travel (beginning state ' 1 '), proceedings entering any failed state are $\mathrm{i}=1,2,13,14,15,16$ taking ' $\varepsilon$ ' $=0$

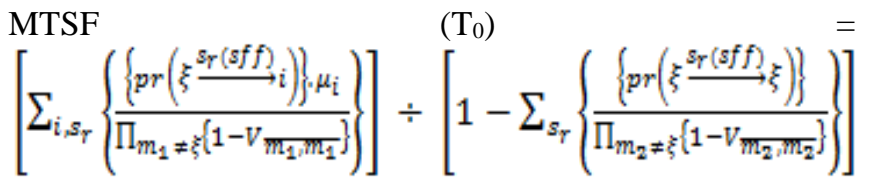

$\mathrm{T}_{0}=\frac{\mathrm{V}_{1,1} \mu_{1}+\mathrm{V}_{1,2} \mu_{2}+\mathrm{V}_{1,13} \mu_{13}+\mathrm{V}_{1,14} \mu_{14}+\mathrm{V}_{1,15} \mu_{15}+\mathrm{V}_{1,16} \mu_{16}}{1-P_{12} \cdot P_{21}}$
AVAILABILITY OF THE SYSTEM (A): From figure 1 the regenerative states at which the framework is accessible are $\mathrm{j}=(1,2,13,14,15,16)$ and $\mathrm{i}=1,2 \ldots 17$ taking ' $\varepsilon^{\prime}$ ' $=0$. $A_{0}=$

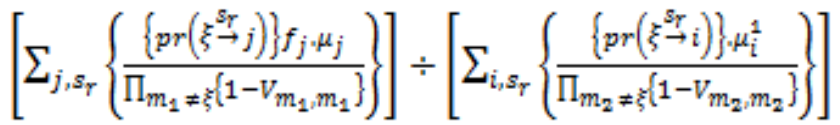
$A_{0}=\left[\Sigma_{j} V_{\xi_{j} j} \cdot f_{j} \times \mu_{j}\right] \div\left[\sum_{i} V_{\xi_{j} i} \times \mu_{i}^{1}\right]$ $A_{0}=\frac{V_{1,1} \mu_{1}+V_{1,2} \mu_{2}+V_{1,13} \mu_{13}+V_{1,14} \mu_{14}+V_{1,15} \mu_{15}+V_{1,16} \mu_{16}}{V_{1,1} \mu_{1}+V_{1,2} \mu_{2}+V_{1,1} \mu_{3}+\ldots \ldots \ldots \ldots \ldots .+V_{1,17} \mu_{17}}$ $\mathrm{A}_{0}=\frac{\mathrm{V}_{1,1} \mu_{1}+\mathrm{V}_{1,2} \mu_{2}+\mathrm{V}_{1,13} \mu_{13}+\mathrm{V}_{1,14} \mu_{14}+\mathrm{V}_{1,15} \mu_{15}+\mathrm{V}_{1,16} \mu_{16}}{D}$ WhereD= $V_{1,1} \mu_{1}+V_{1,2} \mu_{2}+V_{1,1} \mu_{3}+$ $+V_{1,17} \mu_{17}$ 


\section{Behaviour Analysis of Alloy Wheel Plant}

BUSY PERIOD ( $\left.\mathbf{B}_{\mathbf{0}}\right)$ : From figure 1 the regenerative states where server is occupied while doing fixes are $\mathrm{j}=2,3 \ldots 17$ and the regenerative the regenerative state are $i=1,2 \ldots \ldots \ldots$ 17, ' $\varepsilon$ ' $=0$.

$B_{0}=$

$\left[\sum_{j_{2} s_{Y}}\left\{\frac{\left.\left\{p r\left(\xi^{s_{Y}}\right) j\right)\right\} \eta_{j}}{\prod_{m_{1} \neq \xi}\left\{1-V_{m_{1}, m_{1}}\right\}}\right\}\right] \div\left[\sum_{i_{2} s_{Y}}\left\{\frac{\left\{p r\left(\xi^{s_{Y}} \rightarrow i\right)\right\}, p_{i}^{1}}{\prod_{m_{2} \neq \xi}\left\{1-V_{m_{2} / m_{2}}\right\}}\right\}\right.$

$B_{0}=\left[\Sigma_{j} V_{\xi_{j}} \cdot \eta_{j}\right] \div\left[\Sigma_{i} V_{\xi_{j},} \cdot \mu_{i}^{1}\right]$

$\mathrm{B}_{0}=\frac{\mathrm{V}_{1,2} \mu_{1}+\mathrm{V}_{1,3} \mu_{3}+\cdots+\mathrm{V}_{1,14} \mu_{14}+\mathrm{V}_{1,15} \mu_{15}+\mathrm{V}_{1,16} \mu_{16}+\mathrm{V}_{1,17} \mu_{17}}{D}$

EXPECTED NUMBER OF REPAIREMAN ( $\left.\mathrm{V}_{0}\right)$ : From the figure 1 the regenerative states where server visit for fix

Table VI. Mean Times to System Failures

Table VI. Mean Times to System Failures

\begin{tabular}{|l|l|l|l|l|}
\hline & $\beta=0.50$ & $\beta=0.55$ & $\beta=0.60$ & $\beta=0.65$ \\
\hline $\begin{array}{l}\alpha= \\
0.10\end{array}$ & 1.32790 & 1.29882 & 1.23030 & 1.20120 \\
\hline $\begin{array}{l}\alpha= \\
0.15\end{array}$ & 1.12325 & 1.06139 & 1.01455 & 1.01123 \\
\hline $\begin{array}{l}\alpha= \\
0.20\end{array}$ & 0.93581 & 0.81081 & 0.72492 & 0.71232 \\
\hline $\begin{array}{l}\alpha= \\
0.25\end{array}$ & 0.83251 & 0.71235 & 0.63251 & 0.61251 \\
\hline
\end{tabular}

Table VIII. Busy Period of Server

\begin{tabular}{|l|l|l|l|l|}
\hline & $\beta=0.50$ & $\beta=0.55$ & $\beta=0.60$ & $\beta=0.65$ \\
\hline $\begin{array}{l}\alpha= \\
0.10\end{array}$ & 0.11455 & 0.11461 & 0.11477 & 0.11488 \\
\hline $\begin{array}{l}\alpha= \\
0.15\end{array}$ & 0.24813 & 0.24865 & 0.24897 & 0.24901 \\
\hline $\begin{array}{l}\alpha= \\
0.20\end{array}$ & 0.31482 & 0.32267 & 0.32813 & 0.33231 \\
\hline $\begin{array}{l}\alpha= \\
0.25\end{array}$ & 0.42561 & 0.42863 & 0.43125 & 0.43654 \\
\hline
\end{tabular}

Table VII. Availability of the system

Table VII. Availability of the system
\begin{tabular}{|l|l|l|l|l|}
\hline & $\beta=0.50$ & $\beta=0.55$ & $\beta=0.60$ & $\beta=0.65$ \\
\hline $\begin{array}{l}\alpha= \\
0.10\end{array}$ & 0.11904 & 0.11419 & 0.11517 & 0.10914 \\
\hline $\begin{array}{l}\alpha= \\
0.15\end{array}$ & 0.12328 & 0.12204 & 0.12006 & 0.11904 \\
\hline $\begin{array}{l}\alpha= \\
0.20\end{array}$ & 0.14288 & 0.13235 & 0.12499 & 0.11356 \\
\hline $\begin{array}{l}\alpha= \\
0.25\end{array}$ & 0.16298 & 0.15236 & 0.14256 & 0.13265 \\
\hline
\end{tabular}

Retrieval Number: B3104129219/2019@BEIESP
Published By:

Blue Eyes Intelligence Engineering \& Sciences Publication

of the framework are ' $\mathrm{j}$ ' $=1$ and the regenerative states are ' $\mathrm{i}$ ' $=1$ to $17,{ }^{\prime} \xi{ }^{\prime}=0$, the expected number of server visit per unit time is given by

$\mathrm{V} \quad\left[\sum_{\mathbf{j}, \mathbf{s r}}\left\{\frac{\{\mathbf{p r}(\stackrel{\mathrm{Sr}}{\xi \rightarrow \mathbf{j}})\}}{\pi_{\mathbf{m}_{\mathbf{1}} \neq \xi}\left(1-\mathbf{v}_{\mathbf{m}_{\mathbf{1}, \mathbf{m} 1}}\right)}\right\} \div\right.$

$\left[\sum_{\mathbf{i}, \mathbf{s r}}\left\{\frac{\{\mathbf{p r}(\xi \rightarrow \mathbf{s r})\} \boldsymbol{\mu}_{\mathbf{i}}^{\prime}}{\pi_{\mathbf{m}_{2} \neq \xi}\left(1-\mathbf{V}_{\mathbf{m}_{2, \mathbf{m} 2}}\right)}\right\}\right]$

$\mathrm{V}_{0}=$

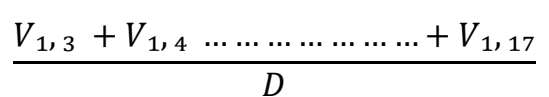

PARTICULAR CASES: $\boldsymbol{\beta}_{\mathrm{i}}=\boldsymbol{\beta}(\mathrm{i}=1,2,3,4,5,6)$

$\boldsymbol{\alpha}_{i}=\boldsymbol{\alpha}(\mathrm{i}=1,2,3,4,5,6,7,8,9,10,11,12$

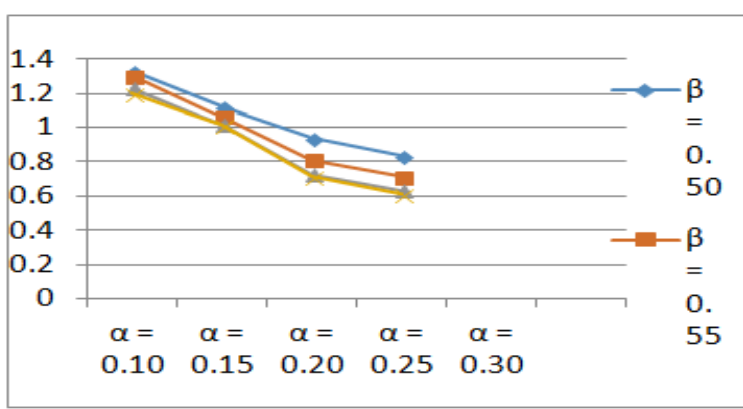

Fig. 2. Mean Times to System Failures

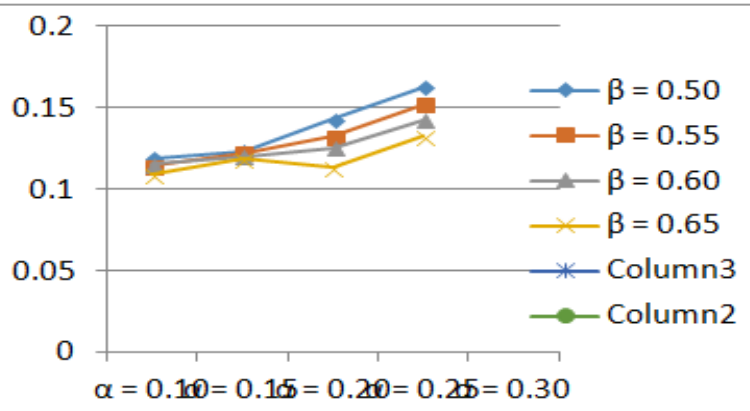

Fig.3. Availability of the system

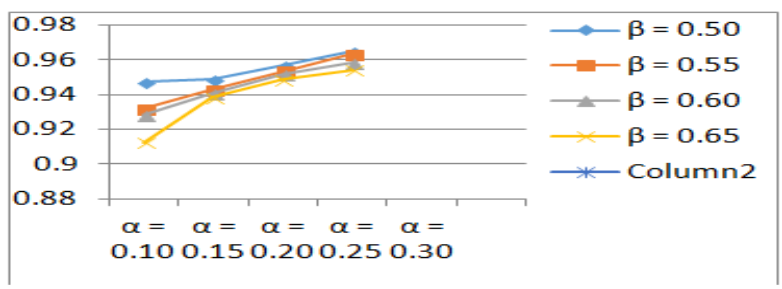

Fig.4. Busy period of Server by the repairman

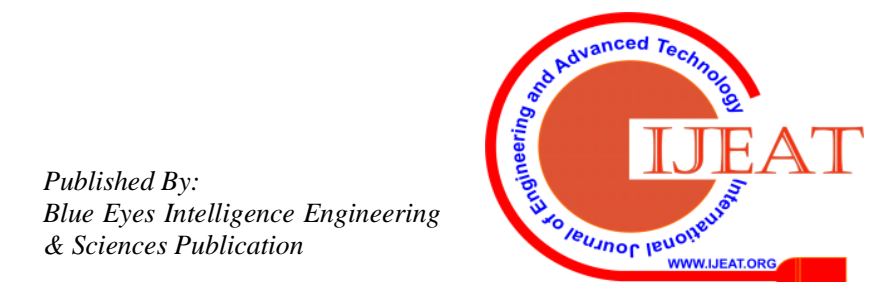


A gander at the table 5 and graph 2 it is obtained that MTSF is large for lower estimations of failure rates of various units subsequently to have longer MTSF failure rates of the considerable number of units ought to be most reduced one for example every one of units must be of good quality and design. A system is ideal if the estimation of $\mathrm{A} 0$ is most elevated for given values failure and repair rates of units. From the above table 6 estimation of $\mathrm{A} 0$ is best corresponding to the most reduced repair rate $\beta$. From the last column it is likewise of the considerable number of units ought to be most reduced one for example every one of units must be of good quality and design. A system is ideal if the estimation of $\mathrm{A} 0$ is most elevated for given values failure and repair rates of units. From the above table 6 estimation of A0 is best corresponding to the most reduced repair rate $\beta$. From the last column it is likewise that there is no significant increment in estimation of $\mathrm{A} 0$ in contrasted with the increase in the repair rates of different units. A framework is said to be ideal if the server's busy period is smallest. From the above table 7 and graph 4 it is seen that busy period values are least if unit's disappointment rate are little as seen from the third Column of the above table. On expending failure rate of units it is watched relating to the expansion in disappointment place of unit ' $\mathrm{P}$ 'the estimation of B0 expands all more quickly contrasted with the failure rate of another unit. From the above table 8, it is seen that the estimation of T0 expands all the more quickly with the expansion in failure rate.

\section{REFERENCE}

1. Kumar, Amit \& Kumar, Vinod \& Modgil, Vikas. (2019). Behavioral study and availability optimization of a multi-state repairable system with hot redundancy. International Journal of Quality \& Reliability Management. 10.1108/IJQRM12-2017-0274.

2. Kumar A., Garg D., Goel P. (2019). Sensitivity Analysis of a Cold Standby System with Priority for Preventive Maintenance. Journal of Advance and Scholarly Researches in Allied Education Vol. 16(4), 253-258.

3. Jyoti Gulati, V. V. Singh and Dilip Kumar Rawal (2018). Performance assessment of two unit's redundant system under different failure and repair policies using copula. International Journal of Reliability and Applications Vol. 19(2) pp. 109-124.

4. Kumar A., Goel P., Garg D., Sahu A. (Redset 2017). System Behavior Analysis in the urea fertilizer industry. Book: Data and Analysis Communications in computer and information Science (CCIS), Springer, Volume 799, Chapter no: 1, pp;3-12.

5. Mangey Ram \& Monika Manglik (2016). Reliability Measures Analysis of an Industrial System under Standby Modes and

\section{AUTHORS PROFILE}

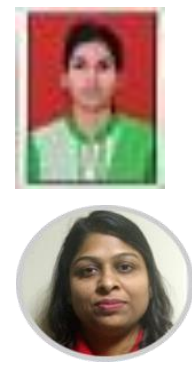

Rajbala ( I am PhD Scholar in

G.d.Goenka University,Gurugram)

Dr. Deepika Garg (Associate

professor inG.D.GoenkaUniv.)
Catastrophic Failure. International Journal of Operations Research and Information Systems Vol. 7(3), pp. 36-37.

6. S.K. Chhillar, A.K. Barak and S.C. Malik (2013). Analysis of a Parallel System with Priority to Repair over Maintenance Subject to Random Shocks. International Journal of Computer Science Issues, Vol. 10(3), pp-317-325.

7. M. Ram, S. B. Singh, and R. G. Varshney 2013. Performance improvement of a parallel redundant system with coverage factor. Journal of Engineering Science and Technology, vol. 8(3), pp. 344350.

8. A. Mehrtash, P. Wang, and L. Goel 2012. Reliability evaluation of power systems considering restructuring and renewable generators. IEEE Transactions on Power Systems, vol. 27 (1), pp. 243-250.

9. T. H. Liu, J. C. Ke, Y. L. Hsu, and Y.-L. Hsu (2011). Bootstrapping computation of availability for a repairable system with standby subject to imperfect switching. Communications in Statistics-Simulation and Computation, vol. 40(4), pp. 469- 483.

10. Wu, C.H. and Zhang, Z.G. (2010). Recent developments in vacation queueing models: a short survey. International Journal of Operations Research, Vol. 7(4), pp. 3-8.

11. Sharma, R. and Sharma, G.C. (2015). Maximum entropy analysis of bulk arrival retrial queue with second optional service and Bernoulli vacation. International Journal of Industrial and Systems Engineering, Vol. 20, pp. 369-396.

12. S.C. and Preeti (2010). Availability analysis of software hardware system with common cause shock failure, spare and switching failure. Journal of International Academy of Physical Sciences, Vol. 14(4), pp. 425-437.

13. M. Nourelfath, M. Fitouhi and M. Machani (2010). An integrated model for production and preventive maintenance planning in multistate systems. IEEE Transactions on Reliability, vol. 59, no. 3, pp. 496506.

14. K. Kumar, J. Singh, and P. Kumar (2009). Fuzzy reliability and fuzzy availability of the serial process in butter- oil processing plant. Journal of Mathematics and Statistics, vol. 5, no. 1, pp. 65-74. 